\section{Human Osteoblast-like Cells Are Permissive for Zika Virus Replication}

\section{To the Editor:}

We read with great interest the letter by Roimicher, et $a l^{1}$. The authors reported a case of a woman with a 4-year diagnosis of rheumatoid arthritis who achieved therapy-induced clinical remission 4 months before acquiring Zika virus (ZIKV) infection. The woman presented with fever, maculopapular rush, conjunctivitis, and arthralgia, and was tested for Chikungunya virus and ZIKV infection. The ZIKV genome was detected in blood and knee synovial fluid (SF). ZIKV viremia become negative after the end of clinical signs (Day 7); however, the patient experienced articular pain about 1 week later, and tested positive for ZIKV RNA in SF, while ZIKV viremia remained negative. These findings characterized for the first time a ZIKV infection localized within the joint of a patient, and raised a more general question about the ZIKV ability to directly target cells of the osteoarticular tissues, thus contributing to the establishment of joint-related symptoms.

Here we provide experimental observation that may support and strengthen the notion of the ability of ZIKV to productively infect cells belonging to the bone lineage. Specifically, we investigated the ability of ZIKV to infect and replicate in human osteoblast-like SV-40 T antigen immortalized cell line (HOBIT; kindly provided by Professor M.C. Re, University of Bologna). For this study, ethical approval was not required, in accordance with the policy of the Italian National Institute for Infectious Diseases L. Spallanzani (Istituto Di Ricovero e Cura a Carattere Scientifico).

HOBIT cells were exposed to either an Asian (ZIKV strain PRVABC59, Genbank KU501215) or an East African (ZIKV strain MR766, Genbank LC002520.1) ZIKV strain for $1 \mathrm{~h}$ at $37^{\circ} \mathrm{C}$, at a multiplicity of infection (MOI) of 0.1 and $1 \mathrm{TCID}_{50} /$ cell. A clear cytopathic effect was observed for both strains starting from $48 \mathrm{~h}$ postinfection (hpi). The production of infectious viral particles was monitored by back-titrating supernatants collected at 6,24, 48,72, and 96 hpi. Table 1 shows a steady increase of ZIKV titer in HOBIT infected with the 2 viral strains at both MOI, thus indicating that these cells are highly permissive to productive infection by ZIKV, releasing up to $10^{7.9} \mathrm{TCID}_{50} / \mathrm{ml}$. The virus yield titer peaks slightly earlier and reaches higher levels with the African strain, suggesting possible differences in the replication kinetics.

Our data extend current knowledge about the broad ZIKV tissue and cell tropism ${ }^{2}$

Indeed, studies on both animal models and human cases reported the presence of ZIKV in skin fibroblasts, epidermal keratinocytes, dendritic cells $^{3}$, human endometrial stromal cells ${ }^{4}$, and cells of the placenta ${ }^{5,6}$. ZIKV also targets neuronal cell types ${ }^{7,8}$ and cells of the reproductive tract, including spermatogonia, Sertoli and Leydig cells, and vaginal epithelium ${ }^{2,9}$. This extensive tropism is paralleled by the detection of ZIKV in multiple body fluids, including tears, saliva, semen, cervical mucus, and urine ${ }^{2,10}$.

Table 1. Kinetics of Asian and African ZIKV replication in HOBIT cells. Supernatants of HOBIT infected with either ZIKV strain at the indicated MOI were collected at the indicated timepoints and back-titrated on Vero E6 cell line by limiting dilution assay. Infectious viral titers were expressed as $\log$ tissue culture infectious dose (TCID) $)_{50} / \mathrm{ml}$.

\begin{tabular}{lcccccc}
\hline ZIKV Strain & \multicolumn{2}{c}{ MOI } & \multicolumn{4}{c}{ Virus Yield $\left(\log \mathrm{TCID}_{50} / \mathrm{ml}\right)$} \\
& & $6 \mathrm{hpi}$ & $24 \mathrm{hpi}$ & $48 \mathrm{hpi}$ & $72 \mathrm{hpi}$ & $96 \mathrm{hpi}$ \\
\hline PRVABC59 (Asian) & 0.1 & $<1.3$ & 3.6 & 5.6 & 6.3 & 6.9 \\
& 1 & 1.9 & 5.3 & 6.2 & 6.8 & 7.3 \\
MR766 (African) & 0.1 & $<1.3$ & 3.8 & 6 & 7.8 & 7.8 \\
& 1 & $<1.3$ & 5.0 & 7.3 & 7.8 & 7.9 \\
\hline
\end{tabular}

ZIKV: Zika virus; HOBIT: human osteoblast-like SV-40 T antigen immortalized cell line; MOI: multiplicity of infection; hpi: hours postinfection.
Overall, the present study, together with the detection of ZIKV in $\mathrm{SF}^{1}$, supports the hypothesis that joint tissue may be a possible reservoir of ZIKV replication, thus expanding the spectrum of ZIKV pathogenesis.

FRANCESCA COLAVITA, Biologist, Laboratory of Virology, National Institute for Infectious Diseases L. Spallanzani, Istituto Di Ricovero e Cura a Carattere Scientifico (IRCCS), Rome; GIUSEPPINA MUSUMECI, Biologist, Department of Microbiology, Section of the Department of Experimental, Diagnostic and Specialty Medicine, School of Medicine, University of Bologna, Bologna; CLAUDIA CAGLIOTI, Biologist, Laboratory of Virology, National Institute for Infectious Diseases L. Spallanzani, IRCCS, Rome, Italy. This study was partly supported by the Italian Ministry of Health (Ricerca Corrente). The authors gratefully acknowledge the scientific support and the mentoring of Dr. Licia Bordi, Dr. Concetta Castilletti, and Dr. Eleonora Lalle (Rome); and the help and encouragement of Dr. Maria Rosaria Capobianchi, Dr. Giuseppe Ippolito (Rome), Prof. Maria Carla Re (Bologna), and Prof. Davide Gibellini (Verona). We also thank Dr. Serena Quartu and Dr. Mirella Biava (Rome) for technical support. Address correspondence to Dr. C. Caglioti, National Institute for Infectious Diseases L. Spallanzani, IRCCS, Laboratory of Virology, Via Portuense 292, Rome, Italy.

E-mail: claudia.caglioti@inmi.it

\section{REFERENCES}

1. Roimicher L, Ferreira OC Jr, Arruda MB, Tanuri A. Zika virus in the joint of a patient with rheumatoid arthritis. J Rheumatol 2017;44:535-6.

2. Miner JJ, Diamond MS. Zika virus pathogenesis and tissue tropism. Cell Host Microbe 2017;21:134-42.

3. Hamel R, Dejarnac O, Wichit S, Ekchariyawat P, Neyret A, Luplertlop N, et al. Biology of Zika virus infection in human skin cells. J Virol 2015;89:8880-96.

4. Pagani I, Ghezzi S, Ulisse A, Rubio A, Turrini F, Garavaglia E, et al. Human endometrial stromal cells are highly permissive to productive infection by zika virus. Sci Rep 2017;7:44286.

5. Bayer A, Lennemann NJ, Ouyang Y, Bramley JC, Morosky S, Marques ET Jr, et al. Type III interferons produced by human placental trophoblasts confer protection against Zika virus infection. Cell Host Microbe 2016;19:705-12.

6. Quicke KM, Bowen JR, Johnson EL, McDonald CE, Ma H, O’Neal JT, et al. Zika virus infects human placental macrophages. Cell Host Microbe 2016;20:83-90.

7. Tang H, Hammack C, Ogden SC, Wen Z, Qian X, Li Y, et al. Zika virus infects human cortical neural progenitors and attenuates their growth. Cell Stem Cell 2016;18:587-90.

8. Li H, Saucedo-Cuevas L, Regla-Nava JA, Chai G, Sheets N, Tang $\mathrm{W}$, et al. Zika virus infects neural progenitors in the adult mouse brain and alters proliferation. Cell Stem Cell 2016;19:593-8.

9. Tang WW, Young MP, Mamidi A, Regla-Nava JA, Kim K, Shresta S. A mouse model of Zika virus sexual transmission and vaginal viral replication. Cell Rep 2016;17:3091-8.

10. Hou W, Armstrong N, Obwolo LA, Thomas M, Pang X, Jones KS, et al. Determination of the cell permissiveness spectrum, mode of RNA replication, and RNA-protein interaction of Zika virus. BMC Infect Dis 2017;17:239.

J Rheumatol 2018;45:3; doi:10.3899/jrheum.170835

$$
\text { Personal non-commercial use only. The Journal of Rheumatology Copyright (C) 2018. All rights reserved. }
$$

